\title{
Successful Treatment of Massive Pulmonary and Venous Thromboembolism with Intravenous Heparin in a Patient with Mild Membranous Nephropathy.
}

\author{
Kunwer Naveed Mukhtar ${ }^{1}$, Shaheen Bibi ${ }^{1}$, Sumbal Nasir Mahmood ${ }^{2}$, Osama \\ Kunwer Naveed ${ }^{3}$
}

${ }^{1}$ Liaquat National Hospital, Karachi, Pakistan.

${ }^{2}$ Ziauddin University Hospital, Karachi, Pakistan.

${ }^{3}$ Dow University of Health Sciences, Karachi, Pakistan.

\begin{abstract}
:
Membranous nephropathy is well described to be associated with hypercoagulable state. Pulmonary embolism is a common occurrence with hypercoagulability and early recognition and prompt treatment including thrombolysis is warranted. We present here a case of massive thrombus in the pulmonary trunk and inferior vena cava in a patient with membranous nephropathy where anticoagulation with heparin was continued with successful resolution over time. We discuss the pros and cons of anticoagulation amangement in such patients and review the literature.
\end{abstract}

Key words: membranous nephropathy, hypercoagulable state, pulmonary embolism, thrombolysis, heparin.

\section{Correspondence To}

Professor Dr Kunwar Naveed Mukhtar

Department of Nephrology

Liaquat National Hospital, Karachi

Email: naveedkunwer@yahoo.com

Received: June 16, 2020. Accepted Dec 3, 2020.

PJKD 2020;4(4):332-35

\section{Introduction}

Membranous nephropathy is the most common cause of nephrotic syndrome after diabetic nephropathy in adults. ${ }^{1}$ Majority of patients presents with nephrotic range proteinuria and occasionally with mild proteinuria. Membranous nephropathy for prognostic and therapeutic purposes is divided into mild, moderate and severe disease. This division is based on amount of proteinuria, presence or absence of renal failure and other complications. ${ }^{2}$

Pulmonary embolism (PE) is one of the dreadful complications of this disease which offers both diagnostic and management challenge. ${ }^{3}$ Early recognition and prompt management can be lifesaving.

Here we are presenting a case of a young male patient who presented with sub-nephrotic range proteinuria and was diagnosed on renal biopsy as post streptococcal glomerulonephritis versus early membranous nephropathy (since electron microscopy is not available) and presented three weeks later with massive pulmonary embolism as well as in inferior vena cava (IVC) and renal vein thrombosis. He was managed with intravenous (IV) unfractionated heparin followed by low molecular weight heparin (LMWH) on discharge. Long term anticoagulation was continued post discharge and resultant clot resolution was seen on contrast enhanced CT pulmonary artery angiogram (CT PA) after 6 months of initial presentation.

\section{Case report:}

21 years old male presented on $30^{\text {th }}$ April 2019, with history of appendectomy 10 days prior to presentation. He now presented with complaint of right sided flank pain and mild right lower quadrant pain for 2 days. On examination he had mild right costovertebral angle tenderness and 1+ edema. Rest of the exam was unremarkable. His initial workup showed a serum creatinine of $1.2 \mathrm{mg} / \mathrm{dl}$ and urine analysis showed 2+ protein and 8-10 RBCs/HPF. Ultrasound abdomen was unremarkable with normal sized kidneys and no stones. A workup for suspected glomerulonephritis was started and serologies were sent. 24-hour urine protein was 2.4 gm, serum albumin was $3.3 \mathrm{~g} / \mathrm{dl}$. A renal biopsy was performed, all immune workup came negative and biopsy was read as resolving post infectious glomerulonephritis with changes suggestive of early membranous nephropathy. Patient presented to the emergency 20 days later with 
sudden onset shortness of breath and profuse sweating. On examination he was cold clammy with a GCS of 14/15 and blood pressure of $85 / 60 \mathrm{~mm}$ of $\mathrm{Hg}$ and pulse of 110 beats/min. He was tachypneic and oxygenation saturation on room air of $85 \%$ which improved to $94 \%$ on 4 liters of Oxygen. Chest examination revealed normal vesicular breath sounds and normal cardiac auscultation. ECG showed depressed PR interval, right heart strain pattern and S1Q3T3, findings suggestive of pulmonary embolism. He had a normal chest radiograph. Complete blood picture (CBC) showed hemoglobin of $15 \mathrm{gm} / \mathrm{dl}$ with slight leukocytosis with white cell count of 12,000/mcl. Patient was shifted to ICU and was stabilized with fluid resuscitation and oxygen. Since suspicion of pulmonary embolism was high therefore patient was started on IV unfractionated heparin, D-dimer was sent, the result of which later came high. Bed side transthoracic echocardiogram showed moderately raised pulmonary artery pressure. After hemodynamic stabilization urgent CT scan whole abdomen and CTPA was done which showed filling defect in both right and left pulmonary arteries extending in both ascending and descending branches of pulmonary trunk suggestive of massive pulmonary embolism (Figure 1A\&B). Minimum pericardial effusion was also seen. Focal areas of filling defects were also seen in inferior vena cava jest beneath the hepatic venous confluence partially extending into right renal veins (Figure $2 \mathrm{~A} \& \mathrm{~B}$ ).

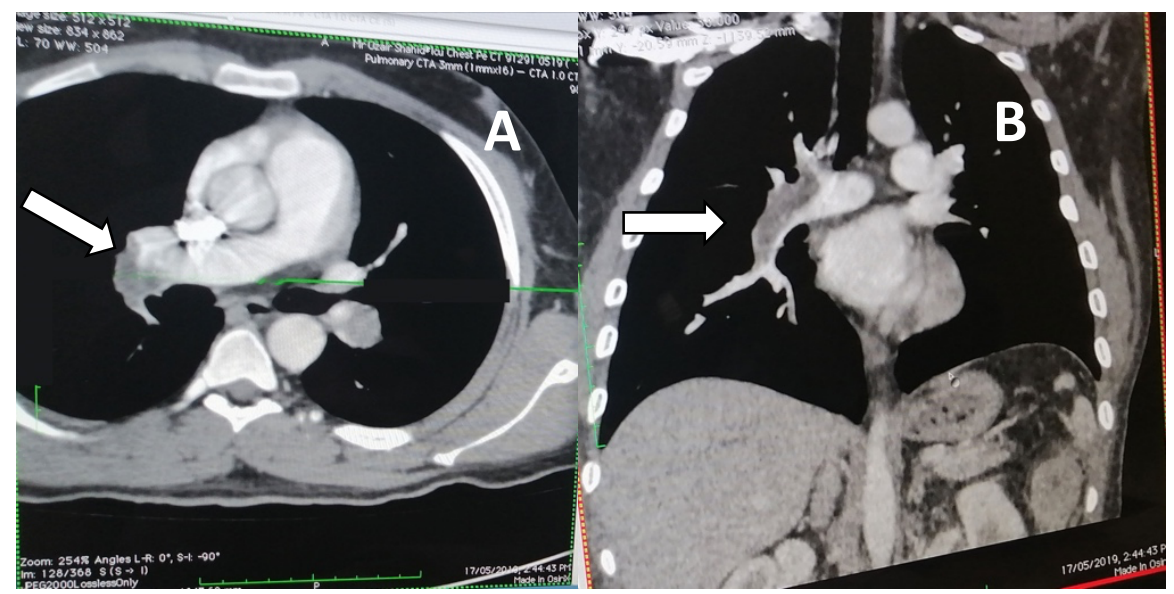

Figure 1: CT Pulmonary angiogram showing filling defect in both right and left pulmonary arteries extending in both ascending and descending branches of pulmonary trunk suggestive of massive pulmonary embolism (A, B).

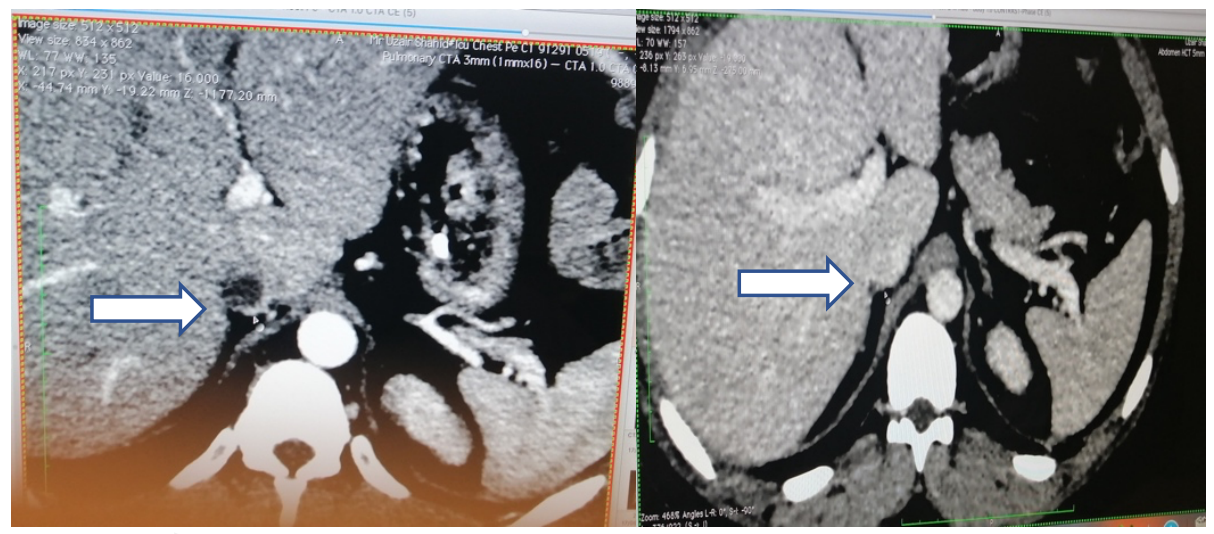

\section{Figure 2: Thrombus seen in the inferior vena cava jest beneath the hepatic venous confluence partially extending into right renal veins $(A, B)$.}

A multidisciplinary meeting was called between vascular surgery, department of pulmonology, cardiothoracic surgery, radiology and nephrology. Possible options as per availability of resources, expertise and cost were all discussed. Thrombolytic therapy was discussed as it is the standard of care but was considered high risk as in our patient thrombus load was high and thrombus in IVC was above renal veins, so IVC filter was deferred due to the risk of dislodgement and further embolism was very high. The surgical thrombectomy option versus thrombolytic therapy was discussed with family but due to high risk of morbidity and mortality family refused.

Patient started to show signs of clinical improvement within 48 hours of heparin infusion and his tachycardia and oxygen saturation started to improve. On day 3 of hospital stay, he got transferred to High dependency unit and was not requiring oxygen. On day 4 he was shifted to ward and got discharged the next day on Low molecular weight heparin (LMWH) at a dose of $60 \mathrm{mg}$ twice a day. He remained on regular follow-up as outpatient and four weeks later repeat CT PA, Figure 3: A\&B, showed much improvement with 
residual thrombosis in left descending pulmonary artery and recanalization of remaining branches of pulmonary artery. There was recanalization in previously noted thrombus in the inferior vena cava, however, there was still a focal filling defect at confluence of right renal vein. Initially no provoking cause for his DVT with PE could be established as all coagulation screen including anti-thrombin III, protein C, protein S, factor V laden, Antiphospholipid antibodies were normal, as was Doppler studies of both lower limbs. His Anti PLA2R which was sent earlier came back positive and patient was diagnosed as a case of idiopathic membranous nephropathy. We continued long term anticoagulation with enoxaparin which was switch to Rivaroxaban after four weeks. Repeat CT scan after six months (Figure 4) showed complete resolution of thrombus of pulmonary, IVC and renal veins.

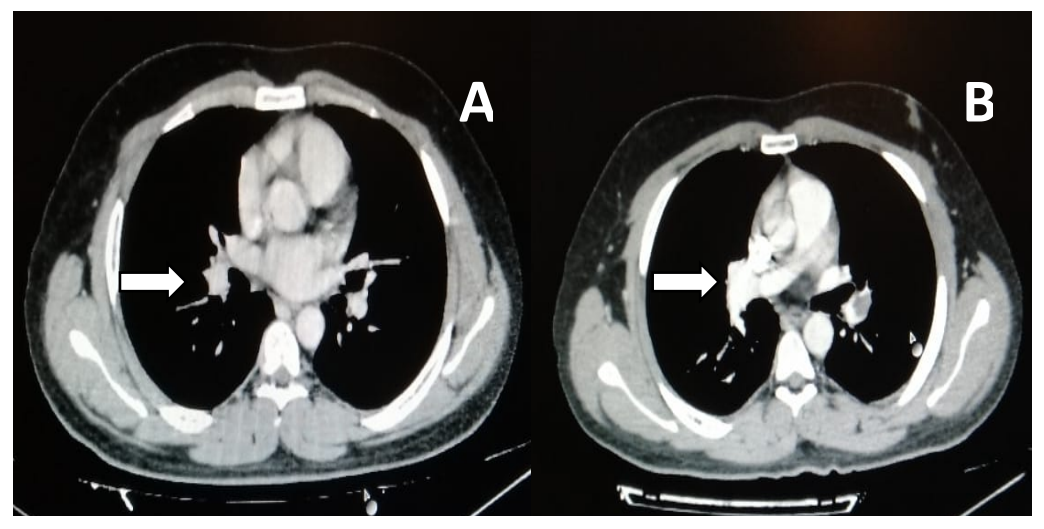

Figure 3: improvement with residual thrombosis in left descending pulmonary artery and recanalization of remaining branches of pulmonary artery (A\&B)

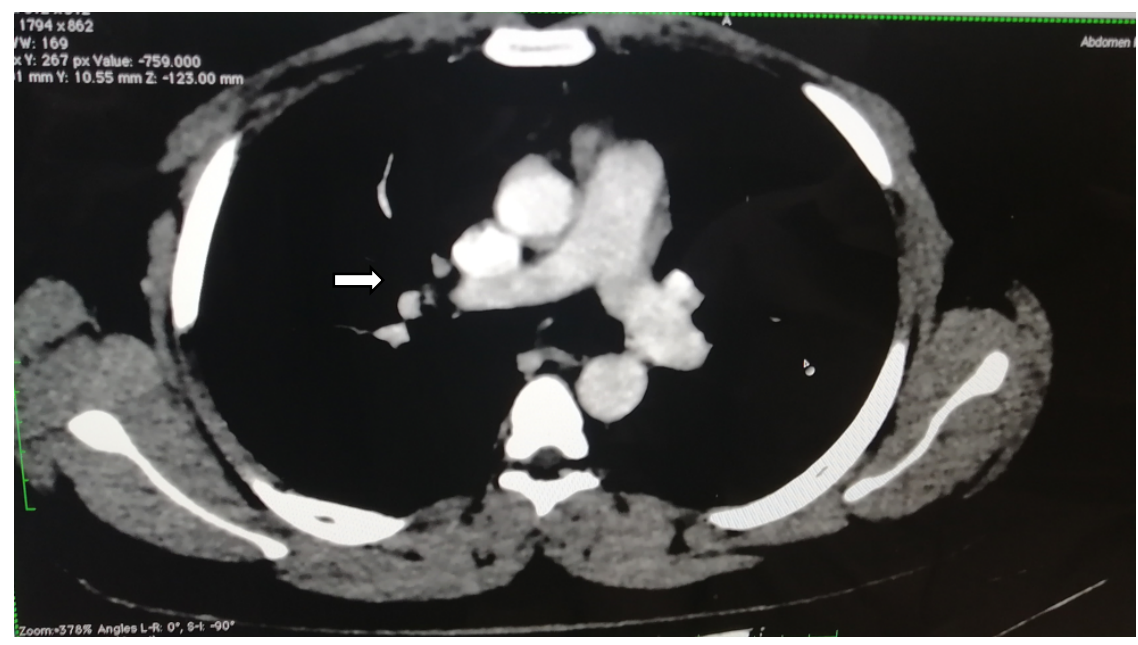

Figure 4: CT scan after six months showed complete resolution of thrombus of pulmonary artery.

\section{Discussion}

We report this unusual case of membranous nephropathy with extensive thrombosis involving both main pulmonary trunks, inferior vena cava and right renal vein thrombosis, who had mild membranous disease by definition. He initially presented with non-nephrotic proteinuria and a serum albumin of $3.3 \mathrm{~g} / \mathrm{dl}$. Usually renal vein thrombosis or pulmonary embolism is found in patients with nephrotic syndrome when serum albumin less than $2.2 \mathrm{gm} / \mathrm{dl}{ }^{4}$ Patients with severe pulmonary embolism are usually treated with thrombolytic therapy either systemic or catheter directed therapy (CDT). ${ }^{5,6}$ In our patient, because of high thrombus load and inability to place IVC filter, he was considered high risk for stroke or worsening of pulmonary embolism. Patient was successfully treated with IV unfractionated heparin/LMWH with resolution of thrombus on follow-up CT scan. There are only few studies comparing between thrombolytic versus heparin use in cases of pulmonary embolism and long term outcome were the same. Konstantinidis et al compared alteplase versus heparin therapy in major pulmonary embolism. ${ }^{7}$ The endpoint of study showed that ventricular strain normalized in both after 1 week and there was no difference in outcome. Similarly, Hennemeyer et.al. in a retrospective study of cases with sub massive and massive PE compared CDT to IV heparin alone showing no difference in 90 days in outcome. ${ }^{8}$ Renal vein thrombosis, either unilateral or bilateral is usually reported with nephrotic syndrome and is treated with IV heparin which is converted to warfarin and keeping target INR of 2.5-3. There is no comparative trial of thrombolytic versus anticoagulation in this patient population, but majority of data agrees with use of IV heparin with good results. The use of thrombolytic is typically reserved in patients with severe renal vein 
MGN and Thromboembolism

thrombosis extending into IVC causing acute renal failure. ${ }^{9,10}$ The risk of bleeding with thrombolytic therapy should be considered, with the potential of renal parenchymal infarction, conversion to hemorrhagic risk is always there in these patients. ${ }^{1}$

The other option was high risk pulmonary surgical embolectomy with high risk of morbidity and mortality and was refused by the patient' s family.

\section{Conclusion}

In conclusion continuous anticoagulation with heparin and later oral anticoagulants may still be beneficial in patients with massive pulmonary thromboembolism where thrombolysis or surgical embolectomy is not possible.

\section{Conflict of interest: None Declared}

\section{References:}

1. Fogo AB, Lusco MA, Najafian B, Alpers CE, AJKD Atlas of Renal pathology: Membranous nephropathy. Am J Kidney Dis. 2015;66(3):e15.

2. Floege J, Barbour SJ, Caltran DC, Hogan JJ, Nachman PH, Tang SCW et. al. Management and treatment of glomerular diseases (part 1): cconcusions from a Kidney Disease: Improving Global Outcomes (KDIGO) Controversies Conference Kidney Int 2019;95(2):268

3. Kasper W, Konstantinides S, Geibel A, Olschewski M, Heinrich F, GrosseKD,et.al. Management strategies and determinants of outcome in acute major pulmonary embolism: results of a multicenter registry. J Am CollCardiol. 1997; 30: 1165 - 1171

4. Llach F Papper S, Massry SG The clinical spectrum of renal vein thrombosis; acute and chronic. Am J Med. 1980;69(6):819.

5. Nils Kucher and Samuel Z. Goldhaber Management of Massive Pulmonary Embolism. Circulation 2005;112:e28 - e32

6. Jaar BG, Kim HS, Samaniego MD, Lund GB, Atta MG. Percutaneous mechanical thrombectomy: a new approach in the treatment of acute renal vein thrombosis. Nephrol Dial Transplant 2002; 17; 1122-5

7. Konstantinides S, Tiede N, Geibel A, Oischewski M, Just H, Kasper W Comparison of alteplase versus heparin for resolution of major pulmonary embolism. Am J Cardiol. 1998;82(8)966

8. Hennemeyer C, Khan A, McGregor H, Moffett C, Woodhead G Outcomes of catheter directed therapy plus anticoagulation versus anticoagulation alone for submassive and massie pulmonary embolism. Am J Med .2019, 132(2); 240.

9. Crowley JP Matarese RA, Quevedo SF, Garella S. Fibrinolytic therapy for bilateral renal vein thrombosis. Arch Intern Med 1984;144;159160.

10. Lam KK, Lui CC, Successful treatment of acute inferior vena cava and unilateral renal vein thrombosis by local infusion of recombinant tissue plasminogen activator. Am J Kidney Dis 1998;32;1075-9.

11. Frontera JA, Lewin JJ 3rd, Rabinstein AA, Aisiku IP, Alexandrov AW, Cook AM, et.al. Guideline for Reversal of Antithrombotics in Intracranial Hemorrhage: A Statement for Healthcare Professionals from the Neurocritical Care Society and Society of Critical Care Medicine. Neurocrit Care. 2016;24(1):6.

12. Osborne ZJ, Rossi P, Aucar J, Dharamsy S, Cook S, Wheatley B. Surgical pulmonary embolectomy in a community hospital. Am J Surg. 2014 Mar;207(3):337-41 